\title{
Security Flaw in Simple Generalized Group-Oriented Cryptosystem Using ElGamal Cryptosystem
}

\author{
Chuan-Ming LI, Tzonelih HWANG \\ Department of Computer Science and Information Engineering, National Cheng Kung University \\ Tainan, Taiwan 701, R.O.C. \\ e-mail: licm@ismail.csie.ncku.edu.tw,hwangtl@ismail.csie.ncku.edu.tw
}

\author{
Narn-Yih LEE \\ Department of Information Management, Southern Taiwan University of Technology \\ Tainan, Taiwan 710, R.O.C. \\ e-mail:nylee@mail.stut.edu.tw
}

Received: April 2005

\begin{abstract}
A generalized group-oriented cryptosystem (GGOC) based on ElGamal cryptosystem was proposed by Yang et al. in 2003. This study shows that if the authorized decryption sets of users are not properly predetermined in Yang et al.'s GGOC, an unauthorized decryption set of users can recover the encrypted message without difficulty. This study also presents an improved protocol to resist such an attack.
\end{abstract}

Key words: generalized group-oriented cryptosystem, access structure, security.

\section{Introduction}

In 1987, Desmedt (Desmedt, 1987) first proposed the concept of group-oriented cryptosystem (GOC) in which an encrypted message is sent to a group of users rather than an individual. Only the qualified subsets of users, called positive access instances, can cooperatively decrypt the message. In contrast, any unqualified subset of users, called negative access instance, is not able to correctly decrypt the ciphertext.

Many GOCs and the variants had been proposed in the literature (Chang and Lee, 1993; Frankel, 1990; Harn et al., 2004; Hwang, 1991; Ingemarsson and Simmons, 1991; Ito et al., 1987; Tsai et al., 1999; Yang et al., 2003; Yoon et al., 2004). Recently Yang et al. (Yang et al., 2003) utilized the ElGamal cryptosystem (ElGamal, 1985) to propose a generalized group-oriented cryptosystem (GGOC). In Yang et al.'s GGOC, the sender can freely determine the positive access instances of the receiving group and encrypts the message for each positive access instance by multiplying the users' public keys. The users in the predetermined positive access instances can cooperate to decrypt the message. Yang et al. claimed that, compared with Tsai et al.'s scheme (Tsai et al., 1999), their 
GGOC performs better in lowering the computational complexity for the sender and no symmetric cryptosystems are used to encrypt/decrypt the message.

This study will show that if the positive access instances are not properly defined in Yang et al.'s scheme, a negative access instance can decrypt the ciphertext without difficulty. This study also presents an improved scheme to avoid this security flaw.

The rest of this study is structured as follows. The next section deals with the preliminaries of the GGOC. Section 3 briefly reviews Yang et al.'s protocol. In Section 4, we demonstrate the security flaw in Yang et al.'s GGOC and then present an improved protocol to avoid this attack. Finally, a short conclusion is given in Section 5.

\section{Preliminaries}

Let $U_{0}$ denotes the sender of a message, and $U=\left\{U_{1}, U_{2}, \ldots, U_{n}\right\}$ denotes a receiving group of $n$ users. In the GGOC, the set of all positive access instances is called the positive access structure which denoted as $F$, and the set of all negative access instances is called the negative access structure which denoted as $W . F$ and $W$ must satisfy following propositions.

Proposition 1. $F \cup W=2^{U}$ and $F \cap W=\phi$, where $2^{U}$ is the power set of $U$.

Proposition 2. If $f$ is a positive access instance, then any subset $f^{\prime}, f \subseteq f^{\prime} \subseteq U$, is also a positive access instance. In other words, a positive access structure should be monotone.

Proposition 3. If $w$ is a negative access instance, then any subset $w^{\prime}, w^{\prime} \subseteq w \subseteq U$, is also a negative access instance.

Definition 1. Let $\operatorname{Min}(F)$ be the minimal set in F, called the minimal access structure. $\operatorname{Min}(F)$ is given as

$$
\operatorname{Min}(F)=\left\{f \in F \mid f^{\prime} \nsubseteq f, \forall f^{\prime} \in F-\{f\}\right\} .
$$

For convenience, a positive access structure will always be given in the minimal set and simply be called an access structure in this paper. Take a group of four users $U=\left\{U_{1}, U_{2}, U_{3}, U_{4}\right\}$, for example. Suppose there exists an access structure $F=$ $\left\{\left\{U_{1}, U_{2}, U_{3}\right\},\left\{U_{2}, U_{3}, U_{4}\right\},\left\{U_{1}, U_{4}\right\}\right\}$ which allows the ciphertext to be decrypted cooperatively either by $U_{1}, U_{2}$ and $U_{3}$ or by $U_{2}, U_{3}$ and $U_{4}$ or by $U_{1}$ and $U_{4}$. The access structure can also be represented in the disjunctive normal form (DNF) as follows

$$
F=f_{1}+f_{2}+f_{3}=U_{1} U_{2} U_{3}+U_{2} U_{3} U_{4}+U_{1} U_{4}
$$

where $f_{1}=U_{1} U_{2} U_{3}, f_{2}=U_{2} U_{3} U_{4}$, and $f_{3}=U_{1} U_{4}$ are positive access instances. 


\section{Review of Yang et al.'s GGOC}

This section briefly reviews Yang et al.'s protocol (Yang et al., 2003) as follows. Assume that $U_{0}$ is the sender of a message $M$, and $U_{1}, U_{2}, \ldots, U_{n}$ are all the users in the receiving group. Let $p$ be a large prime such that $p-1$ has a large prime factor $q$. Each user $U_{i}$, for $i=1,2, \ldots, n$, in the system has a private key $x_{i}, x_{i} \in G F(p)$, and the corresponding public key

$$
y_{i}=g^{x_{i}} \quad(\bmod p)
$$

where $g$ is a generator of order $q$ in $G F(p)$. To send the message $M$ to the receiving group, $U_{0}$ firstly determines the access structure $F=f_{1}+f_{2}+\ldots+f_{k}$, and then performs the following steps.

Step 1. Choose a random number $r, r \in G F(p)$, and compute $R=g^{r} \quad(\bmod p)$.

Step 2. Compute $C_{j}=M \cdot\left(\prod_{U_{i} \in f_{j}} y_{i}\right)^{r}(\bmod p)$, for $j=1,2, \ldots, k$.

Step 3. Send $\left\{F, R, C_{1}, C_{2}, \ldots, C_{k}\right\}$ to the receiving group.

After receiving $\left\{F, R, C_{1}, C_{2}, \ldots, C_{k}\right\}$ from the sender, the users in the access instance $f_{j}\left(f_{j}=U_{j_{1}} U_{j_{2}} \cdots U_{j_{v}}\right)$ can cooperate to decrypt the message by using their secret keys as follows.

Step 1. Compute $t_{i}=R^{x_{i}}(\bmod p)$, for $i=j_{1}, j_{2}, \ldots, j_{v}$.

Step 2. Recover $M=C_{j} \cdot\left(\prod_{U_{i} \in f_{j}} t_{i}\right)^{-1}(\bmod p)$.

In Yang et al.'s protocol, the sender encrypts the message by multiplying the users' public keys $y_{i}$ to be the public key in the original ElGamal cryptosystem. Then, the users $U_{i}$ in the positive access instance $f_{j}$ can cooperate to decrypt the message. According to the above descriptions, if each access instance has only one single user, Yang et al.'s GGOC is exactly the same as the ElGamal cryptosystem.

\section{Attack on Yang et al.'s GGOC and the Improvement}

\subsection{The Security Flaw in Yang et al.'s GGOC}

Yang et al. claimed that their GGOC is more efficient than Tsai et al.'s scheme (Tsai et al., 1999) in terms of sender's computational complexity and no symmetric cryptosystems are used. However, we find that if the access structure is not properly defined, a negative access instance is able to decrypt the ciphertext. Now, we first use an example to demonstrate this security flaw.

Let the access structure $F$ be predetermined by $U_{0}$ as

$$
F=f_{1}+f_{2}+f_{3}=U_{1} U_{2} U_{3}+U_{2} U_{3} U_{4}+U_{1} U_{4}
$$

$U_{0}$ chooses a random number $r \in G F(p)$, and then computes $R=g^{r} \quad(\bmod p)$ and the ciphertext $\left\{C_{1}, C_{2}, C_{3}\right\}$ as 


$$
\begin{aligned}
& C_{1}=M \cdot\left(y_{1} \cdot y_{2} \cdot y_{3}\right)^{r} \quad(\bmod p), \\
& C_{2}=M \cdot\left(y_{2} \cdot y_{3} \cdot y_{4}\right)^{r} \quad(\bmod p), \\
& C_{3}=M \cdot\left(y_{1} \cdot y_{4}\right)^{r} \quad(\bmod p) .
\end{aligned}
$$

$U_{0}$ sends $\left\{F, R, C_{1}, C_{2}, C_{3}\right\}$ to the receiving group. Obviously, the ciphertext can be legally decrypted either by $U_{1}, U_{2}$ and $U_{3}$ or by $U_{2}, U_{3}$ and $U_{4}$ or by $U_{1}$ and $U_{4}$. However, although $U_{1}$ is not allowed to decrypt the ciphertext alone, he/she can calculate that

$$
\begin{aligned}
\left(C_{1} / C_{2}\right) \cdot C_{3} & =\left(\frac{M \cdot\left(y_{1} \cdot y_{2} \cdot y_{3}\right)^{r}}{M \cdot\left(y_{2} \cdot y_{3} \cdot y_{4}\right)^{r}}\right) \cdot\left(M \cdot\left(y_{1} \cdot y_{4}\right)^{r}\right) \\
& =M \cdot y_{1}^{2 r} \\
& =M \cdot R^{2 x_{1}} \quad(\bmod p) .
\end{aligned}
$$

Thus, $U_{1}$ can easily decrypt the ciphertext alone by computing

$$
M=\left(\left(C_{1} / C_{2}\right) \cdot C_{3}\right) \cdot R^{-2 x_{1}} \quad(\bmod p) .
$$

According to the above example, we generalize the security flaw in Yang et al.'s GGOC as follows. Let $F=f_{1}+f_{2}+\ldots+f_{k}$ be the access structure. Suppose $f_{a}$, $f_{b}$ and $f_{c}$, where $a, b, c \in\{1,2, \ldots, k\}$, are positive access instances in $F$ and

$$
f_{a}=\left(f_{b} \cup f_{c}\right)-\left(f_{b} \cap f_{c}\right) .
$$

Let the sender of a message $M$ send the ciphertext $\left\{F, R, C_{1}, C_{2}, \ldots, C_{k}\right\}$ to the receiving group. A negative access instance $f_{h}^{\prime}$, where $f_{h}^{\prime}=f_{a} \cap f_{b}$, can recover the message $M$ by computing

$$
M=\left(\left(C_{b} / C_{c}\right) \cdot C_{a}\right) \cdot\left(\prod_{U_{i} \in f_{h}^{\prime}} R^{-2 x_{i}}\right) \quad(\bmod p) .
$$

\subsection{Improvement}

Since the ciphertext $C_{j}$, for $j=1,2, \ldots, k$, in Yang et al.'s GGOC is the product of the message $M$ and $\left(\prod_{U_{i} \in f_{j}} y_{i}\right)^{r}$, a negative access instance $f_{h}^{\prime}$ can utilize the multiplication and division of ciphertexts to eliminate some unknown factors in $C_{j}$. Hence, any method which prevents the negative access instances from eliminating the unknown factors in the ciphertext $C_{j}$ can overcome the security flaw. One possible solution is that, instead 
of computing the product of the message $M$ and $\left(\prod_{U_{i} \in f_{j}} y_{i}\right)^{r}$, the sender calculates the ciphertexts $C_{j}$ as

$$
C_{j}=M \oplus\left(\prod_{U_{i} \in f_{j}} y_{i}\right)^{r} \quad(\bmod p),
$$

where $\oplus$ denotes the bit-wise exclusive OR operation. To decrypt the ciphertext, the users in the access instance $f_{j}\left(f_{j}=U_{j_{1}} U_{j_{2}} \cdots U_{j_{v}}\right)$ compute $t_{i}=R^{x_{i}} \quad(\bmod p)$, for $i=j_{1}, j_{2}, \ldots, j_{v}$, and recover the message $M=C_{j} \oplus\left(\prod_{U_{i} \in f_{j}} t_{i}\right) \quad(\bmod p)$.

The only difference between the improvement and Yang et al.'s GGOC is the calculation of the ciphertext $C_{j}$, as the improvement uses XOR operation rather than the multiplication. Thus, the security of the improvement is also based on the ElGamel cryptosystem, which in turn is based on the difficulty of solving the discrete logarithm problem. It is difficult for a malicious user to compute the secret key $x_{i}$ of user $U_{i}$ from the public key $y_{i}=g^{x_{i}} \quad(\bmod p)$. It is also difficult for an adversary to obtain the random number $r$ from the equation $R=g^{r} \quad(\bmod p)$. Besides, it is very difficult for the legal users $U_{i}$ (for $i=j_{1}, j_{2}, \ldots, j_{v}$ ) in the access instance $f_{j}$ to disclose the secret keys $x_{k}$ of other users $U_{k}$ from the equation $t_{k}=R^{x_{k}} \quad(\bmod p)\left(\right.$ for $k \neq i$ and $\left.k=j_{1}, j_{2}, \ldots, j_{v}\right)$. On the other hand, to recover $M$ from the message $\left\{F, R, C_{1}, C_{2}, \ldots, C_{k}\right\}$ sent by $U_{0}$, the malicious user has to either break the Diffie-Hellman scheme (Diffie and Hellman, 1976) or find all the terms $t_{i}, i \in f_{j}$. Moreover, unlike Yang et al.'s GGOC, a negative access instance $f_{h}^{\prime}$ in the improvement cannot utilize the multiplication or division of ciphertexts to eliminate the unknown factors in the ciphertexts $C_{j}$. Thus, the improvement is secure against the security flaw proposed in this study.

\section{Conclusions}

This study demonstrates a security flaw in Yang et al.'s GGOC, and fixes the protocol to avoid such an attack.

\section{Acknowledgments}

The authors thank Mr. Sheng-Wei Liao for helpful discussions. The authors also thank the National Science Council of the Republic of China, Taiwan for financially supporting this research under Contract NO. NSC 95-2221-E-218-030.

\section{References}

Chang, C.-C., and H.-C. Lee (1993). A new generalized group-oriented cryptoscheme without trusted centers. IEEE Journal on Selected Areas in Communications, 11(5), 725-729. 
Desmedt, Y. (1987). Society and group oriented cryptography: a new concept. In Advances in Cryptology: Proceedings of Crypto'87, LNCS. pp. 120-127.

Diffie, W., and M.E. Hellman (1976). New directions in cryptography. IEEE Transactions on Information Theory, 22(6), 644-654.

ElGamal, T. (1985). A public-key cryptosystem and a signature scheme based on discrete logarithms. IEEE Transactions on Information Theory, IT31, 469-472.

Frankel, Y. (1990). A practical protocol for large group oriented networks. In Advances in Cryptology: Proceedings of Eurocrypto'89, LNCS. pp. 55-61.

Harn, L., C.-Y. Lin and T.-C. Wu (2004). Structured multisignature algorithms. In IEE Proceedings - Computers and Digital Techniques, 151(3). pp. 231-234.

Hwang, T. (1991) Cryptosystem for group oriented cryptography. In Advances in Cryptology: Proceedings of Eurocrypto'90, LNCS. pp. 352-360.

Ingemarsson, I., and G.J. Simmons (1991). A protocol to set up shared secret schemes without the assistance of a mutually trusted party. In Advances in Cryptology: Proceedings of Eurocrypto'90, LNCS. pp. 266-282.

Ito, M., A. Saito and T. Nishizeki (1987). Secret sharing schemes realizing general access structure. In Proc. IEEE Global Telecommunication Conf., Globecom'87. IEEE Press, Piscat-away, New Jersey. pp. 99-102.

Tsai, J.-J., T. Hwang and C.-H. Wang (1999). New generalized group-oriented cryptosystem based on diffiehellman scheme. Computer Communications, 22(8), 727-729.

Yang, C.-C., T.-T. Chang, J.-W. Li and M.-S. Hwang (2003). Simple generalized group-oriented cryptosystem using elgamal cryptosystem. INFORMATICA, 14(1), 111-120.

Yoon, E.-J., E.-K. Ryu and K.-Y. Yoo (2004). Efficient remote user authentication scheme based on generalized ElGamal signature scheme. IEEE Transactions on Consumer Electronics, 50(2), 568-570.

C.-M. Li was born in Tainan Taiwan, in 1964. He received the MS degree in computer science from National Cheng Kung University in 1994. He is currently a PhD student at Department of Computer and Information Engineering, Nation Cheng Kung University. His research interests include data security and cryptography.

T. Hwang was born in Tainan Taiwan, in 1958. He received his undergraduate degree in National Cheng Kung University in 1980, and the MS and PhD degrees in computer science from the University of Southwestern, Louisiana, USA, in 1988. He is presently a professor of Department of Computer and Information Engineering, Nation Cheng Kung University. His research interests include cryptology, network security and coding theory.

N.-Y. Lee was born in Chiayi, Taiwan, in 1967. He received the BS degree in information science from Tunghi University in 1990 and the MS degree in applied mathematics from Chung-Hsing University in 1992 and the $\mathrm{PhD}$ degree in information engineering from National Cheng-Kung University in 1996 . He is currently a professor in the Information Management Department, Southern Taiwan University of Technology, Tainan, Taiwan. His research interests in information security, cryptography, and smart card system.

\title{
Nesudètingos apibendrintosios grupinès kriptografinès sistemos, naudojančios EIGamalio kriptografinę sistemą, apsaugos trūkumas
}

\author{
Chuan-Ming LI, Tzonelih HWANG, Narn-Yih LEE
}

Yang ir kiti 2003 m. pasiūlè apibendrintają grupinę kriptografinę sistemą (GGOC), kurios pagrindą sudaro ElGamalio algoritmas. Straipsnyje parodyta, kad jei igaliojimų suteikimo vartotojams aprašai nėra tinkamai apibrež̌ti GGOC sistemoje, tuomet neigaliotieji vartotojai gali užšifruota pranešimą nesunkiai dešifruoti. Pasiūlytas geresnis protokolas, kuris apsaugo nuo tokios pranešimo dešifravimo atakos. 